\title{
An Intelligent Prediction System of Students Academic Performance based on Deep Learning and FPSOPCNN
}

\author{
E.M.N. Sharmila
}

\begin{abstract}
This paper proposes a new method based on text extraction techniques for predicting student outcomes using cognitive computation. Predicting student academic achievement is most helpful in helping educators and learners improve their teaching and learning processes. This shows that these students have different experiences that influence their level of information capture in the classroom as they have the potential to use different lenses for training. This document provides a predictive examination of student academic performance in Tamil Nadu College in India during the academic year 2018 and 2019. First, this work applies statistical examination to gain insights from the data. Then, two datasets were obtained. The first dataset contains variables obtained before the beginning of the school year and the second includes study variables collected two months after the beginning of the semester. Convolution Neural Network and Fuzzy Particle Swarm Optimization Pulse Coupled Neural Network (FPSOPCNN) are designed to predict the end-of-year student performance for each dataset. .

Keywords : Cognitive Computing, Efficient Prediction System; Educational Data Mining; Learning Analytics model; CNN; FPSOCNN .
\end{abstract}

\section{INTRODUCTION}

Educational data processing (ADM) and analytical learning (ELA) are two prominent areas that integrate critical applications of computing knowledge in education. And training. The two areas are not separated by any solid boundaries, and instead, they often share common goals and stakeholders. However, they differ greatly in many dimensions, including the method of study. These differences were correctly explained by Siemens and Baker (2012). In short, people play a central role in LA, while much of the EDM work is done through automation. Humans play a role in the EDM, but this is a component of the Ls as they are a key component of the device. In addition, the LA model and analyzes the entire training system as opposed to the EDM break and overcome methods.

ADM is inspired by advances in data mining and machine learning. The overall goal is to help teachers, students and other stakeholders reach their respective goals through the use

Revised Manuscript Received on April 25, 2020.

* Correspondence Author

E.M.N Sharmila*, Arts and Crafts Instructor, Alagappa University College of Education, Alagappa University, Karaikudi Email: emnsharmila@gmail.com

(C) The Authors. Published by Blue Eyes Intelligence Engineering and Sciences Publication (BEIESP). This is an open access article under the CC BY-NC-ND license (http://creativecommons.org/licenses/by-nc-nd/4.0/) of big data in education. This relies heavily on understanding and modeling the way students learn. As a result, the International Data Mining Association (2016) defines the sector as "concerned with the development of more and more unique data mining methods that come from education sites and use these methods. Get better. The understanding and limitations of the students they study. "It should be noted here that this society holds an annual international conference on data collection for education that began in 2008. Predicting student outcomes based on a variety of factors is a popular thread for ADM Research.

Arriving at educational institutions, improving student models so that student characteristics or outcomes can be predicted very well in advance is a key area of EDM applications. As a result, many researchers have begun exploring different techniques of data extraction to help teachers or instructors evaluate and refine their course organization [7]. Predicting student performance is the worst in our current education systems. If student performance is predicted very much in advance, it can maintain or improve the quality of education by predicting students' substantive interests, student-level activities, and helping to improve their performance in school universities and educational institutes. the classification of drop-off points can also be done by this [4]. With the help of machine learning techniques in conjunction with ADM, a continuous evaluation system is implemented by a number of institutions today. These schemes are useful for improving student performance. Using full-time students is a key motto of the Continuous Assessment System. Pretending pipelines and data exchange are the result of an effort to spread machine learning strategies. They contribute to the presentation of data to provide active machine learning and focus on the shortcomings of existing learning algorithms [1]. Studies have been found in some of the deep learning programs that can be applied to a variety of subjects, such as image processing, natural language processing, and object detection[8].

To predict student outcomes, knowledge discovery is requested here to dig into the rules of the dataset of the learning management system. Methods for in-depth training and data extraction are used here. The main contributions of the report are summarized as follows:

1. To develop a brilliant optimization approach named CUCKOOPCNN for features selection process.

2. To develop a deep learning approach named Improved Convolution Neural Network (CNN) for feature classification.

3. To develop efficient student performance prediction

Published By:

Blue Eyes Intelligence Engineering

\& Sciences Publication

10.35940/ijeat.D6621.049420 


\section{An Intelligent Prediction System of Students Academic Performance based on Deep Learning and FPSOPCNN}

system provide the best recommendation to the teacher.

4. To attain high prediction accuracy than the other existing approaches

The paper remains are prepared as follows. Section 2 reviews your work. The rules for initial deployment and time for placing a virtual machine in the data center are proposed in Part III. Section IV describes the experimental results using a model simulation model. The conclusion as well as the future work of this study are presented in Section V.

\section{RELATED WORK}

The download of EDM education data is now widespread due to the increasing availability of electronic resources, the use of online and online educational tools. Numerous studies are being conducted to use the best educational tools and technologies [3]. Using EDM techniques to predict or analyze student outcomes and improve students who fall into the classroom, a model of neural networks has been developed that can be useful for both students and teachers in exploring the big data available in the field. Education [12]. Emotional examination was conducted to understand students' different learning styles and their learning plans to improve teaching [14].

Student behavior characteristics were examined with other characteristics, and a model was proposed based on data mining techniques, which provided a high accuracy of $22.1 \%$ after removing the behaviors. In addition, a $25.8 \%$ increase in accuracy was found using the assembly method [5]. A total of 473 case studies found that $70 \%$ accuracy came from the Bayesian classifier. The naive Bayes classifier, ANN, KNN, and j48 were used to classify dropouts. $87 \%$ and $79.7 \%$ accuracy was obtained from the nearest neighbor $\mathrm{K}$, and the tree decided to perform a 10-fold cross-examination [10]. SVM divides the class into large courtyards, with skyscrapers (15). Data extraction techniques, such as logistic regression and multidimensionality, produce exceptional results in health-related data [16].

The best methods are used by decision trees to set or achieve a specific goal in the real world [13]. Combining this concept with increasing training time [9] explains the widespread use in ADMs. A decision tree was used to predict the passage or failure of a class in the 15,150 datasets, 85.92\% were achieved [11].

\section{PROPOSED METHOD}

In this work, we propose a efficient student performance prediction system. To do this, this work introduce one efficient prediction algorithm based on and FPSOPCNN and convolutional neural network (CNN). The outline of our proposed work is shown in Fig.1. The proposed work consists of four modules. They are

1. Data Preprocessing

2. Attribute Selection

3. Feature Extraction

4. Prediction Model Generation

\section{A. Data Preprocessing}

Initially, student records were collected from the university's enterprise database. The data is then reformatted at the data conversion stage to prepare for the following algorithm. In the data cleaning process, the parameters used in the survey are set and the missing data is removed or filled in with a zero value.

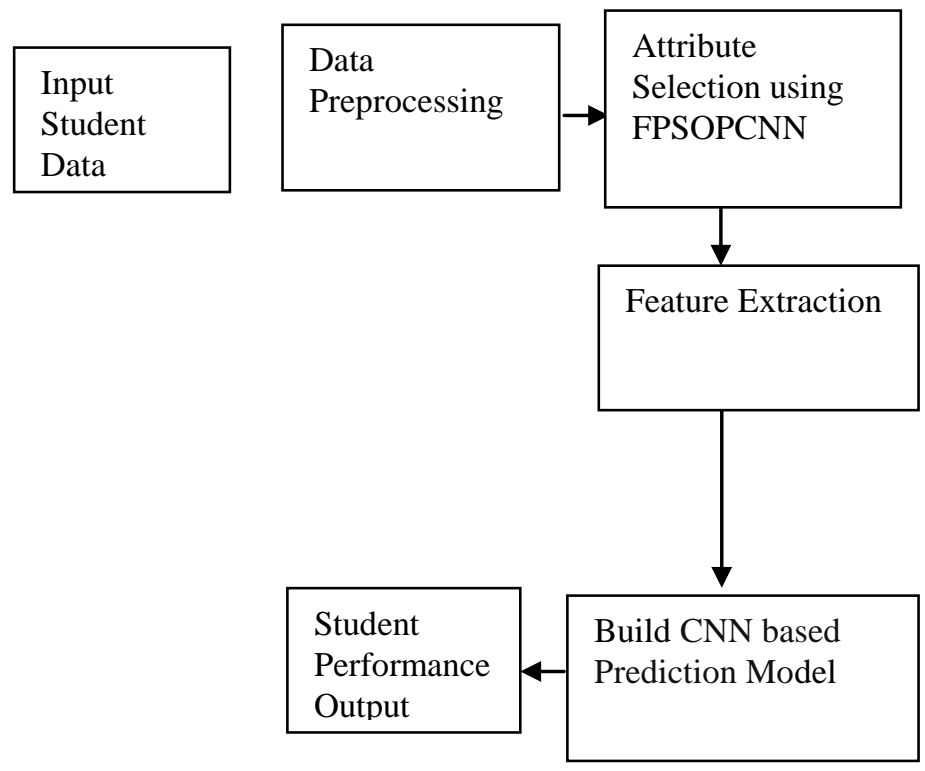

Fig.1 Outline of the Proposed Work

\section{B. Attribute Selection}

In attribute selection, the most important attributes of the student database are only selected by using the novel FPSOPCNN attribute selection approach.

\section{Fuzzy Particle Swarm Optimization}

To get good forecast results, several types of functions are performed at the same time. Because different types of features may contain complementary information, this may lead to better efficiency predictions by selecting discriminant features from different trait spaces. The advantage of feature selection is to define the meaning of the original feature set.

Fuzzy Particle Swam Optimization (FPSO) is used to select features. FPSO [20] has a knowledge base that incorporates information provided by experts in the form of ambiguous rules for managing complex interface languages, effectively converting pure data into an ambiguous set of preferences. Which uses them in conjunction with a knowledge base to draw conclusions using reasoning methods and pointers Infusion or rendered so vague physical activity monitoring using the infusion method. The FPSO is based on the following equation

$$
\text { FPSO }(\mathrm{p})=\sum_{\mathrm{k}=1}^{\mathrm{n}} \sum_{\mathrm{i}=1}^{\mathrm{c}}\left[\tau_{\mathrm{ik}}\right]^{\mathrm{m}}\left\|\mathrm{y}_{\mathrm{k}}-\mathrm{W}_{\mathrm{i}}\right\|^{2}
$$

where, $m>1$ is a real number, $W_{i}$ is the cluster centre of $i, y_{k}$ is the vector part of $\mathrm{k}$.

$$
W_{i}=\frac{\sum_{k=1}^{\mathrm{n}}\left[\tau_{\mathrm{ik}}\right]^{\mathrm{m}} \mathrm{y}_{\mathrm{k}}}{\sum_{\mathrm{k}=1}^{\mathrm{n}}\left[\tau_{\mathrm{ik}}\right]^{\mathrm{m}}}
$$

$$
\left[\tau_{i k}\right]^{(t+1)}=\left[\sum_{j=1}^{c}\left(\frac{\left\|y_{k}-w_{i}^{(t)}\right\|^{2}}{\left\|y_{k}-w_{i}^{(t)}\right\|^{2}}\right)^{\frac{1}{m-1}}\right]^{-1}
$$

Blue Eyes Intelligence Engineering \& Sciences Publication (c) Copyriaht: All riahts reserved.

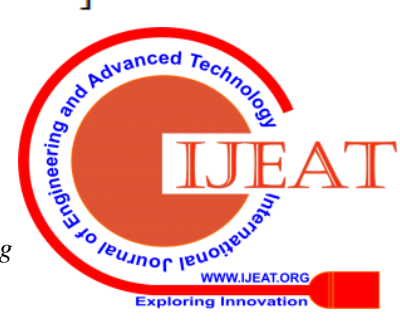


Where c $=1,2, \ldots \mathrm{n},\left\|\mathrm{y}_{\mathbf{k}}-\mathrm{W}_{\mathrm{i}}\right\|^{2}$ represents the Euclidean distance between $y_{k}$ and $W_{i}$, and $\left[\tau_{i k}\right]^{(t+1)}$ is the membership degree of part $\mathrm{k}$ in group $\mathrm{i}$.

Fuzzy Particle Swarm Optimization Pulse Coupled Neural Network (FPSOPCNN)

The disadvantage of the FPSO algorithm is that it is easy to access the best localities in a large space and has low convergence in the process. To overcome the disadvantages of the FPSO, it is combined with the PCCN.

Push-up neural networks are a self-organizing network model that requires no training, and this network mimics the activity of the visual cortex of mammals. A PCNN can be a two-dimensional, one-sided network of integrators and firing with 1: 1 data connections. The neuron has an input, connection, and pulse. The feeding and attachment sections provide input signals to the neuron. From the receptor region, the main entrance is the feeder entrance. The receptor region contains consecutive data of the corresponding region in the input. The input is the next input of the lateral connection to the adjacent neuron. The difference between these studies in evolutionary programs. The implication is that the power connection has a slower response time than the power connection. The standard PCNN model is described as generating the following equation

$\mathrm{Sij}$ is the input stimulus such as the best values from FPSO Fij[n] - Feedback input of the neuron in (i,j)

Lij[n] - Linking item

$\mathrm{F}_{\mathrm{ij}}[\mathrm{n}]=$

$\mathrm{e}^{-a_{F}} \mathrm{Fij}[\mathrm{n}-1]+\mathrm{V}_{\mathrm{F}} \quad \sum_{\mathrm{K}, \mathrm{I}} \mathrm{W}_{\mathrm{i}, \mathrm{j}, \mathrm{K}, \mathrm{I}} \mathrm{Y}_{\mathrm{ij}}[\mathrm{n}-1]+$

$\mathrm{S}_{\text {ij }}$

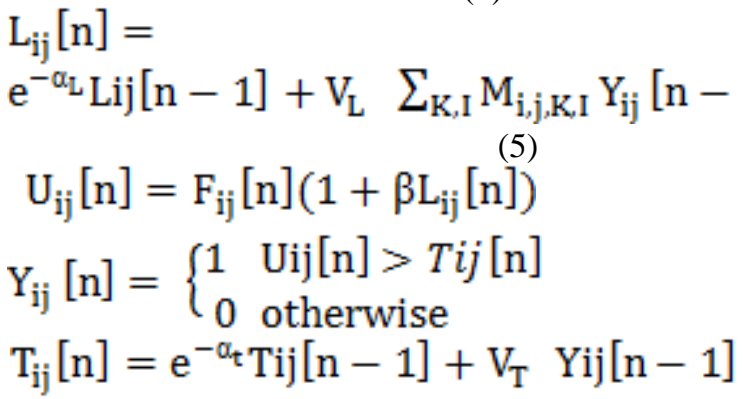

\section{Feature Extraction}

After selected the important attributes, the next step is to generate the feature matrix. In this step three feature matrices are generated namely grade matrix, performance matrix, interest matrix based on the students mark, performance and interests.

The recommendation system has three basic conditions that are user, element and rating. The task of the recommendation is to predict the rating the user will provide for all the priceless objects, and then guide the user to the highest predicted result. Similarly, in the grading management system (GMS) , there are three subjects: student, course, and assessment / evaluation. In this setting, the task is to guess the course markers the student has not learned. There is a similar match between the GMS student sample and the recommended system, so the course and assessment / evaluation students will be user-defined and evaluated \{student or consumer; Courses $\rightarrow$ Subjects; Rating $\rightarrow$ rating\}. Like a similar mapping \{Student user; Courses $\rightarrow$ Subjects; Performance $\rightarrow$ class , \{ student-user; Courses $\rightarrow$ Subjects; Context information $\rightarrow$ classes $\}$ and \{students-users; Courses $\rightarrow$ Subjects; Interest $\rightarrow$ Rating $\}$ is created.

\section{Prediction Model Generation}

Convolutional neural networks are similar to normal neural networks. They are composed of neutrons with weight, training, and bias. Each neuron receives some input, executes a point product, and prefers to follow it indirectly. $\mathrm{CNN}$ has one or more layers and interconnected layers. The integrated layer is also called the test layer. Normally CNN Used for classification purposes. In this work CNN is used to predict student's performance. The pool layer was used to apply the model downstream. It is used to reduce computation time by reducing the features extracted in the light layer, as shown in Fig 2.

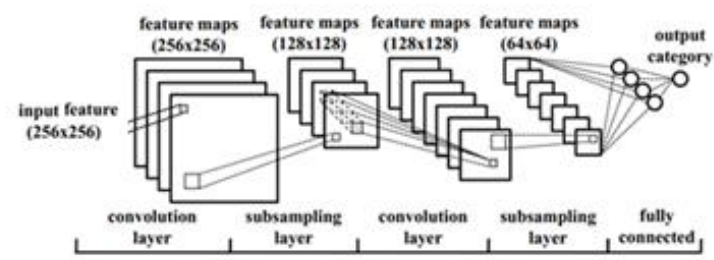

Fig 2 Prediction Model Generation using CNN

There are two types of pools: Max Pool and Average Pool. In the maximum concentration, the value of the largest pixel is considered in the receiver field of the filter. In the pool, the average of all values is considered in the receiving field. The result of the pool layer is given as input into the next solution layer.

\section{RESULT AND EXAMINATION}

\section{A. Data Used}

Datasets of student can be collected from various Universities like UCI, Cleveland, etc. for our recommendation system . The student dataset contains 64 attributes are in total. From that only 44 attributes are selected. The attributes like Tsubjects, XSL, Pusubjects, PUSL, Group, Tboard, TAVG, PU-Average, PUboard, InterestedSubject, interested-profession, multiple-attempts, medium, english-proficiency, Gender, knowledge-level, cognitive-skill,courses,English,Maths,Hindi,French-FL,LL,S ansrit,

Commerce,Economics,Computers,EnvironmentalScience, $\mathrm{Ar}$ t,Physics,Chemistry,Biology,Electronics,History,Geography, Sociology,Politicalscience,Psychology,Botony,Zoology,Ho mescience,Businessmaths,Statistics and Accountacy etcthe title or heads unless they are unavoidable.

\section{B. Units}

Several performance indicators are available to evaluate the effectiveness of the student performance prediction process. The used parameters are shown in following section.

\section{Detection Accuracy}

Published By:

Blue Eyes Intelligence Engineering

\& Sciences Publication

.35940/ijeat.D6621.049426

(C) Copyright: All rights reserved. 
Accuracy Detection is a measurement system that measures the degree of closeness between the original outputs and the correct prediction outputs.

\section{Error Rate}

$$
\text { Accuracy }=\frac{\mathrm{TP}+\mathrm{TN}}{\mathrm{TP}+\mathrm{FP}+\mathrm{TN}+\mathrm{FN}}(9)
$$

The error rate is a measurement system that measures incorrectly labeled outputs that generate input data that give students results.

$$
\text { Error Rate }=\frac{\text { No of Images of Falsely predicted results }}{\text { Total No of results }}
$$

\section{Precision Rate}

PCNN

FPSOPCNN

$$
82.74
$$

80.84

$$
85.11
$$

83.13
79.97

91.23

As can be seen from Table 1, the accuracy of the FPSOPCNN is in the range 93-97, which is higher than the other methods. Therefore, the FPSOPCNN is considered best for prediction model. Figure 8 depicts the accuracy of the FPSOPCNN findings.

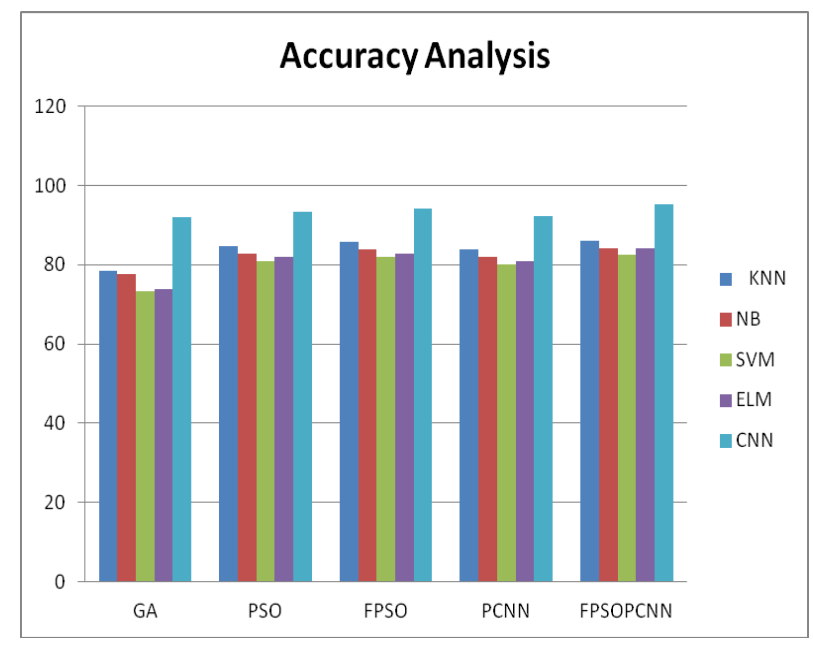

\section{Precision Rate Examination of Proposed Prediction Model}

In this experiment, the FPSOPCNN will be evaluated which are used in the work. Table 2 lists the FPSOPCNN precision examination.

Table 2: Precision Rate Examination of Proposed Prediction Model

\section{F-Measure}

$$
\text { Specificity }=\frac{\mathrm{TN}}{(\mathrm{FP}+\mathrm{TN})}(14)
$$

The F measure is the proportion of the product of accuracy in recalling the amount of precision and recall.

$$
\mathrm{F}_{\mathrm{m}}=(1+\alpha) * \frac{\text { Precision } * \text { Recall }}{\omega *(\text { Precision } * \text { Recall })}(15)
$$

\section{Accuracy Examination of Proposed Prediction Model}

\begin{tabular}{|c|c|c|c|c|c|}
\hline Classifier & & & & & \\
\hline Metrics & KNN & NB & SVM & ELM & CNN \\
\hline GA & 77.34 & 76.72 & 72.29 & 72.79 & 91.11 \\
\hline PSO & 83.75 & 81.85 & 79.95 & 80.98 & 92.24 \\
\hline
\end{tabular}

In this experiment, the FPSOPCNN will be evaluated which are used in the work. Table 1 lists the FPSOPCNN accuracy analyzes.

Table 1: Detection Accuracy examination of Proposed Prediction Model

FPSO

$\begin{array}{lllll}84.66 & 82.76 \quad 80.86 & 81.89 & 93.15\end{array}$

\begin{tabular}{lrrrrr}
\hline Metrics & & NB & SVM & ELM & CNN \\
& KNN & & & & \\
\hline GA & 78.67 & 78.05 & 73.62 & 74.12 & 92.44 \\
PSO & 85.08 & 83.18 & 81.28 & 82.31 & 93.57 \\
& & & & & \\
FPSO & 85.99 & 84.09 & 82.19 & 83.22 & 94.48 \\
PCNN & 84.07 & 82.17 & 80.27 & 81.3 & 92.56 \\
& & & & & \\
FPSOPCNN & 86.44 & 84.46 & 82.71 & 84.36 & 95.56 \\
& & & & & \\
\hline
\end{tabular}

As can be seen from Table 2, the Precision of the FPSOPCNN is in the range 92-96, which is higher than the other methods. Therefore, the FPSOPCNN is considered best for prediction model. Figure 8 depicts the Precision of the FPSOPCNN findings.

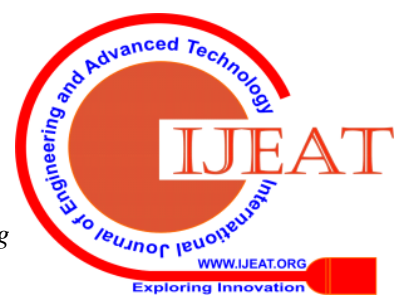




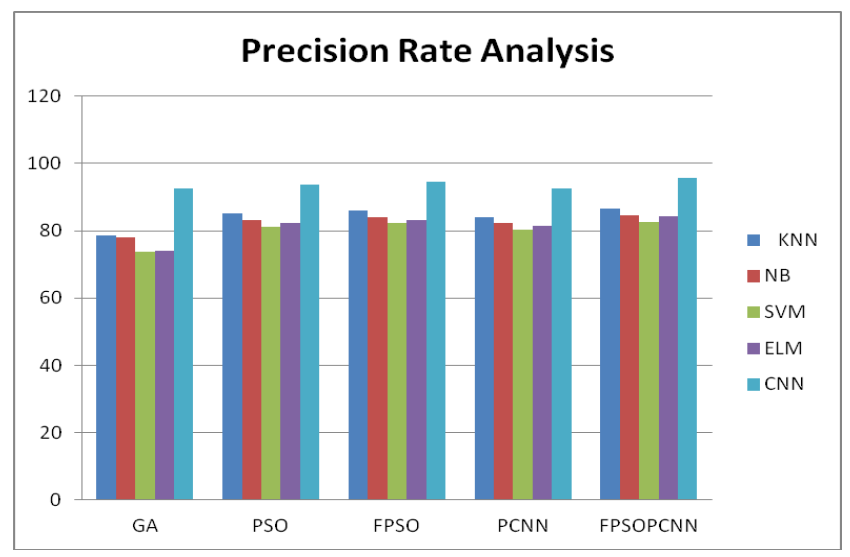

4.3.3 Recall Rate Examination of Proposed Prediction Model

In this experiment, the FPSOPCNN will be evaluated which are used in the work. Table 3 lists the FPSOPCNN recall examination.

Table 3: Recall Rate Examination of Proposed Prediction Model

\section{Classifier}

\begin{tabular}{lrlrrr}
\hline Metrics & & NB & SVM & ELM & CNN \\
& KNN & & & & \\
\hline GA & 76.65 & 76.03 & 71.6 & 72.1 & 90.42 \\
PSO & 83.06 & 81.16 & 79.26 & 80.29 & 91.55 \\
& & & & & \\
& 83.97 & 82.07 & 80.17 & 81.2 & 92.46 \\
FPSO & & & & & \\
PCNN & 82.05 & 80.15 & 78.25 & 79.28 & 90.54 \\
FPSOPCNN & 84.42 & 82.44 & 80.69 & 82.34 & 93.54 \\
& & & & & \\
\hline
\end{tabular}

As can be seen from Table 3, the recall of the FPSOPCNN is in the range 90-93, which is higher than the other methods. Therefore, the FPSOPCNN is considered best for prediction model. Figure 8 depicts the recall of the FPSOPCNN findings.

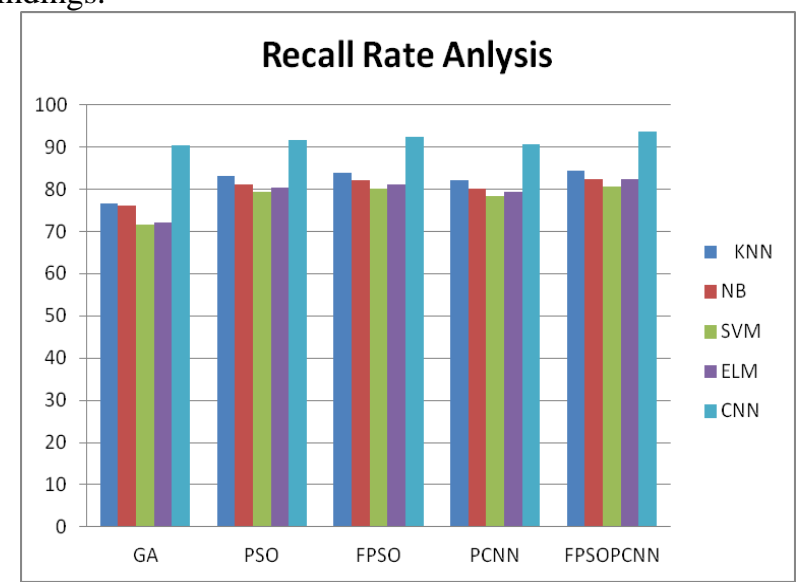

Sensitivity Examination of Proposed Prediction Model
In this experiment, the FPSOPCNN will be evaluated which are used in the work. Table 4 lists the FPSOPCNN sensitivity examination.

As can be seen from Table 4, the sensitivity of the FPSOPCNN is in the range 91-95, which is higher than the other methods. Therefore, the FPSOPCNN classifier is considered best for prediction model. Figure 8 depicts the sensitivity of the FPSOPCNN findings.

Table 4: Sensitivity Examination of Proposed Prediction Model

Classifier

\begin{tabular}{lrrrrr}
\hline Metrics & \multicolumn{2}{l}{ NB } & SVM & ELM & CNN \\
& KNN & & & & \\
\hline GA & 78.16 & 77.54 & 73.11 & 73.61 & 91.93 \\
PSO & 84.57 & 82.67 & 80.77 & 81.8 & 93.06 \\
& & & & & \\
FPSO & 85.48 & 83.58 & 81.68 & 82.71 & 93.97 \\
PCNN & 83.56 & 81.66 & 79.76 & 80.79 & 92.05 \\
& & & & & \\
FPSOPCNN & 85.93 & 83.95 & 82.2 & 83.85 & 95.05 \\
& & & & & \\
\hline
\end{tabular}

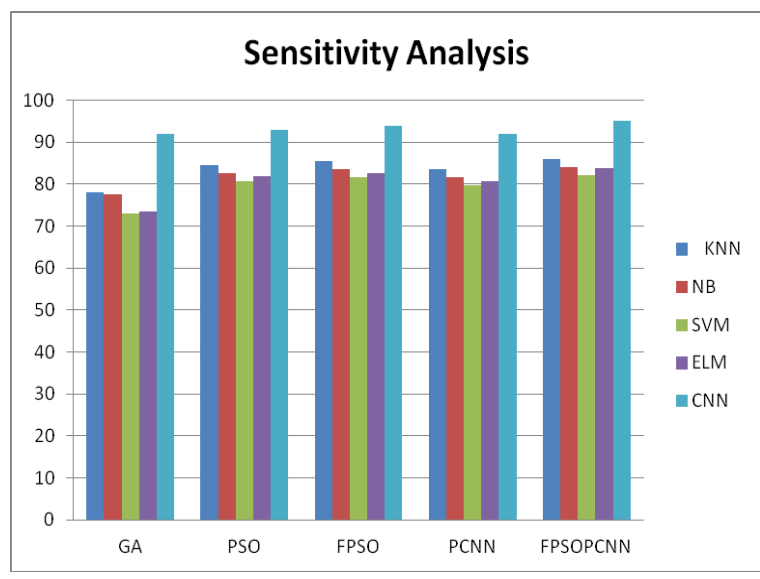

Specificity Examination of Proposed Prediction Model

In this experiment, the FPSOPCNN will be evaluated which are used in the work. Table 5 lists the FPSOPCNN specificity examination.
Published By:

Blue Eyes Intelligence Engineering \& Sciences Publication 
Table 5: Specificity Examination of Proposed Prediction Model

\begin{tabular}{|c|c|c|c|c|c|}
\hline Classifier & & & & & \\
\hline \multirow[t]{2}{*}{ Metrics } & \multirow[b]{2}{*}{ KNN } & \multirow[t]{2}{*}{ NB } & \multirow{2}{*}{$M^{S V}$} & \multirow{2}{*}{ EL } & \multirow{2}{*}{$\mathrm{N}^{\mathrm{CN}}$} \\
\hline & & & & & \\
\hline GA & 78. & 77. & 73. & 73. & 91. \\
\hline & 18 & 56 & 13 & 63 & 95 \\
\hline PSO & $\begin{array}{r}84 . \\
59\end{array}$ & $\begin{array}{r}82 . \\
69\end{array}$ & $\begin{array}{r}80 . \\
79\end{array}$ & $\begin{array}{r}81 . \\
82\end{array}$ & $\begin{array}{r}93 . \\
08\end{array}$ \\
\hline & 85. & 83. & 81. & 82. & 93. \\
\hline FPSO & 5 & 6 & 7 & 73 & 99 \\
\hline PCNN & $\begin{array}{r}83 . \\
58\end{array}$ & $\begin{array}{r}81 . \\
68\end{array}$ & $\begin{array}{r}79 . \\
78\end{array}$ & $\begin{array}{r}80 . \\
81\end{array}$ & $\begin{array}{r}92 . \\
07\end{array}$ \\
\hline FPSOPCN & 85. & 83. & 82. & 83. & 95. \\
\hline $\mathrm{N}$ & 95 & 97 & 22 & 87 & 07 \\
\hline
\end{tabular}

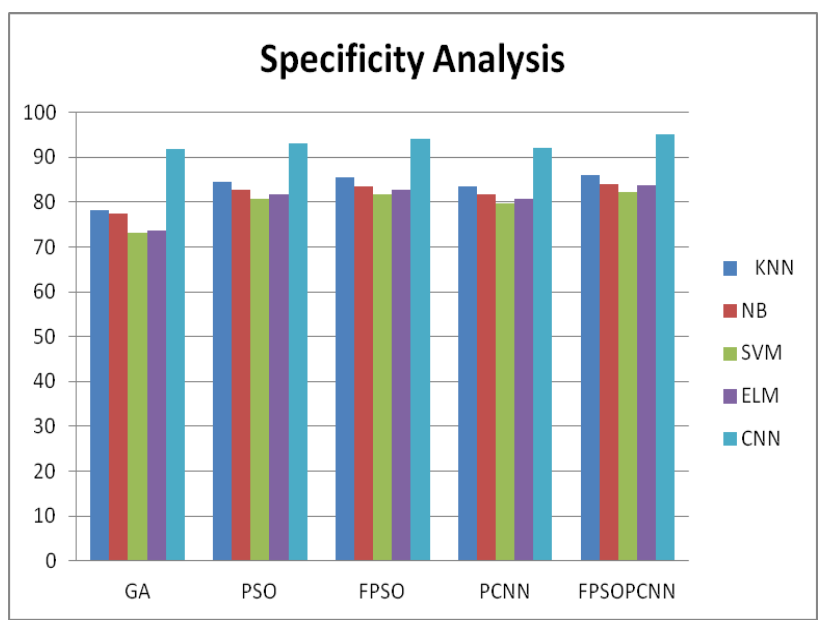

F-Measure Examination of Proposed Prediction Model

Table 6: F-Measure Examination of Proposed Prediction Model

Classifier

\begin{tabular}{lrrrrr}
\hline Metrics & \multicolumn{2}{c}{ NB } & SV & EL & CN \\
& KNN & \multicolumn{2}{c}{ M } & N \\
\hline GA & 76. & 76. & 71. & 72. & 90. \\
& 77 & 15 & 72 & 22 & 54 \\
PSO & 83. & 81. & 79. & 80. & 91. \\
& 18 & 28 & 38 & 41 & 67 \\
& 84. & 82. & 80. & 81. & 92. \\
FPSO & 09 & 19 & 29 & 32 & 58 \\
PCNN & 82. & 80. & 78. & 79. & 90. \\
& 17 & 27 & 37 & 4 & 66 \\
FPSOPCN & 84. & 82. & 80. & 82. & 93. \\
N & 54 & 56 & 81 & 46 & 66 \\
\hline
\end{tabular}

In this experiment, the FPSOPCNN will be evaluated which are used in the work. Table 2 lists the FPSOPCNN F-score examination.

As can be seen from Table 6, the F-Measure of the FPSOPCNN is in the range 90-93, which is higher than the other methods. Therefore, the FPSOPCNN classifier is considered best for prediction model. Figure 8 depicts the F-Measure of the FPSOPCNN findings.

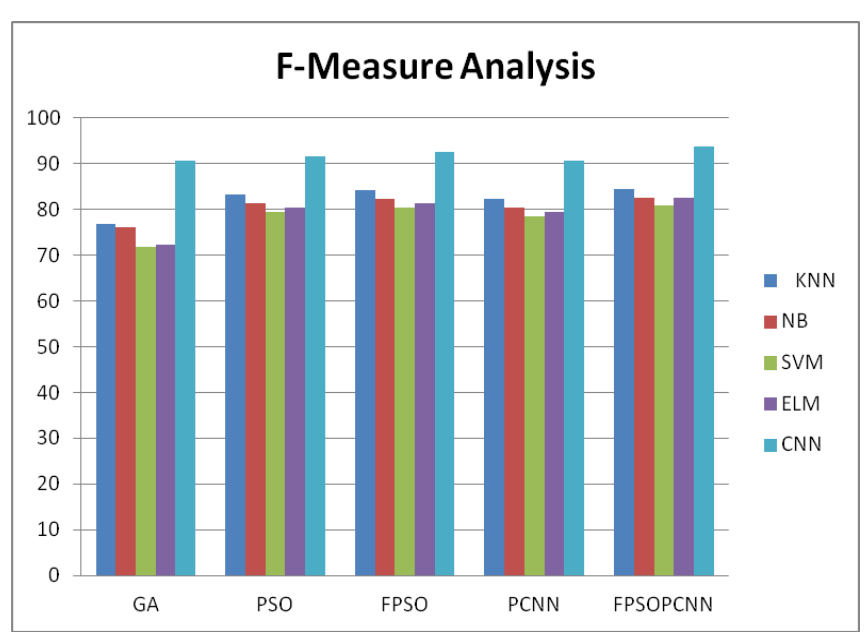

\section{CONCLUSION}

This research examines the effectiveness of students using ADM. The rankings are designed to predict students in different classes, such as high, medium and low. Classifiers used is CNN for classifying students whether they belong to upper or lower middle class. The results of data mining techniques and in-depth studies were compared based on accuracy and precision. It has been found and found that the rankings applied by CNN with FPSOPCNN s more effective than other classification groups, which can be seen with accuracy and precision. Based on, CNN with FPSOPCNN results with the FPSOPCNN technique is more effective than other techniques for predicting student performance.

\section{REFERENCES}

1. Schmidhuber J., "Deep learning in neural networks: An overview". Volume.61, pp.85-117, 2015.

2. Han et al, "Data Mining Concepts and Techniques", 3rd edition, 2011.

3. Pena A, "Educational data mining: A survey and a data miningbased examination of recent works", Exp. sys. app.,vol.41 (4), 1432-1462, 2014.

4. Oyerinde et al, "Predicting Students' Academic PerformancesA Learning Analytics Approach using Multiple Linear Regression, International Journal of Computer Applications", Volume.157, pp.37-44, 2017.

5. Amrieh et al, "Mining Educational Data to Predict Student's academic Performance using Ensemble Methods, International Journal of Database Theory and Application", Vol.9, is.8, pp.119-136, 2016.

6. Scheuer et al.,"Educational data mining. In Encyclopedia of the sciences of learning",pp. 1075-1079, 2012.

7. Romero et al, "Educational data mining: a review of the state of the art.", IEEE Tran. Sys, volume. 0 (6), pp. 601-618, 2010.

8. Sultana et al. "An Extensive Survey on Some Deep Learning Applications", SSRNe-journal, 2018.

9. Rokach et al, "Data mining with decision trees: theory and applications". Wor.sci. 2014.

\section{Published By:}

Blue Eyes Intelligence Engineering \& Sciences Publication

(C) Copyriaht: All rights reserved.

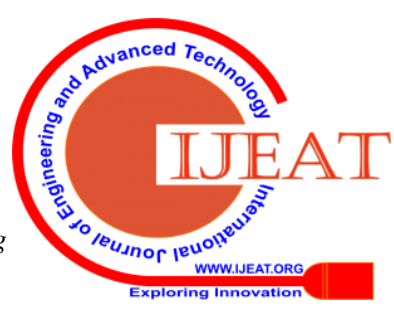


10. Yukselturku et al. "Predicting dropout student: an application of data mining methods in an online education program". Eur. Jour. Dist. Lear., vol.17(1),pp.118- 133, 2014.

11. Jayaprakash et al., "Early alert of academically at-risk students: An open source analytics" init. Jour. Lear. Anal., vol.1(1), pp. 6-47,2014.

12. Livieris, et al. "Predicting students performance using artificial neural networks", pp.321-328, 2012.

13. Baker et al. "Educational data mining and learning analytics. In Learning analytics", pp. 61-75, 2014.

14. Sultana et al. "Prediction on Educational Data based on Deep Learning Approach", .IEEE, 2018.

15. Meyer, D. "Support vector machines the interface to libsvm in package", 2014.

16. Sultana et al, "Predicting Breast Cancer using Support vector Machine and Multi-classifiers", Int. Jour. Eng. Tech., Volume. 7. No.4 20, pp.22-26, 2018.

17. Drigas et al, "Artificial intelligence in special Education: A decade review", Int. Jour. Eng. Edu., 28, 6, 1366- 1372. 2012.

18. Woolf et al, "AI grand challenges for education", Int. Lear. Tech., AI Magazine, 1-22.

19. Teach Thought Staff, "10 roles for artificial intelligence in education",

20. Woolf, B. P., "AI and education: celebrating 30 years of marriage", AIED Work. Proc., 4, 38-47. 2015.

21. Borge, N., "White paper- artificial intelligence to improve education/learning challenges", Inter. Jour. Adv. Eng. Inn. Tech., 2, 6, 10-13. 2016.

22. Duzhin, et al, "Machine learning based app for self-evaluation of teacher-specific instructional style and tools", Ed. Sci. Jour. in MDPI, 8, 7, 1- 15. 2018.

23. Long, $Y$ et al, "Skill diaries: improve student learning in an intelligent tutoring system with periodic self assessment", Art. Int. Edu, 7926, 249-258. 2013

\section{AUTHORS PROFILE}

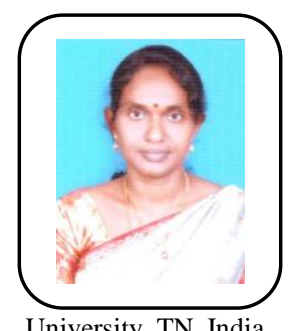

University, TN, India.
Mrs. E.M.N. Sharmila is an Arts and Crafts Instructor at Alagappa University College of Education, Alagappa University, TN, India. Basically she is interested in Computer Science field. Now, she is a computer Science research scholar, TN, India and her research is focused on Computer Networking. Her research interests include Cloud Computing and AI. She did BCA at Madras University and MCA at Annamalai 